\title{
Manipulation with Spin Ordering in Ferromagnetic Semiconductors
}

\author{
Tomasz Dietl \\ Institute of Physics, Polish Academy of Sciences, Al. Lotników 32/46, PL-02668, Warszawa, Poland
}

Received on 10 March, 2003

\begin{abstract}
A short overview is given of recent advances in the field of carrier-controlled ferromagnetism in Mn-based III-V and II-VI diluted magnetic semiconductors and their nanostructures. The tailoring of domain structures and magnetic anisotropy by strain engineering and confinement is described. Experiments demonstrating the tunability of $T_{C}$ by light and electric field are presented.
\end{abstract}

\section{Introduction}

Today's research on spin electronics involves virtually all material families, the most mature being studies on magnetic metal multilayers, in which spin-dependent scattering and tunnelling are being successfully applied in reading heads of high density hard-discs and in magnetic random access memories (MRAM). However, in the context of spintronics [1] particularly interesting are ferromagnetic semiconductors, which combine complementary functionalities of ferromagnetic and semiconductor material systems. One of the relevant questions is to what extent the powerful methods developed to control the carrier concentration and spin polarization in semiconductor quantum structures could serve to tailor the magnitude and orientation of magnetization produced by the spins localized on the magnetic ions. Another important issue concerns the elaboration of methods of injecting and transporting spin currents. In addition of consisting the important ingredient of field sensors and magnetic transistors, spin injection can serve as a tool for fast modulation of light polarization in semiconductors lasers. Indeed, already the early studies of $\mathrm{Cr}$ spinels as well as of rock-salt Eu- [2] and Mn-based [3] chalcogenides led to the observation of a number of outstanding phenomena associated with the interplay between ferromagnetic cooperative phenomena and semiconducting properties. The discovery of ferromagnetism in Mn-based zinc-blende III-V compounds [4], followed by the prediction [5] and observation of ferromagnetism in p-type II-VI materials [6,7] allows one to explore the physics of previously not available combinations of quantum structures and magnetism in semiconductors [8]. This aspect of ferromagnetic semiconductors will be outlined here together with a description of models trying to explain the nature of ferromagnetism in these materials.

\section{Carrier-controlled ferromagnetism}

It is well established that Mn is divalent in II-VI compounds, and assumes the high spin $\mathrm{d}^{5}$ configuration characterized by $S=5 / 2$ and $g=2.0$. Here, $\mathrm{Mn}$ ions neither introduce nor bind carriers, but give rise to the presence of the localized spins. For low carrier densities, II-VI DMS are paramagnetic but neighbor Mn-Mn pairs are antiferromagnetically blocked owing to short-range superexchange interactions. However, this antiferromagnetic coupling can be overcompensated by ferromagnetic interactions mediated by band holes [5-7]. In III-V compounds, in turn, the Mn atom, when substituting a trivalent metal, may assume either of two configurations: (i) $\mathrm{d}^{4}$ or (ii) $\mathrm{d}^{5}$ plus a weakly bound hole, $\mathrm{d}^{5}+\mathrm{h}$. It is now commonly accepted that the Mn impurities act as effective mass acceptors $\left(d^{5}+h\right)$ in the case of antimonides and arsenides, so that they supply both localized spins and holes, a picture supported by MCD [9] and EPR [10] measurements. Just like in other doped semiconductors, if the average distance between the Mn acceptors becomes smaller than $2.5 a_{B}$, where $a_{B}$ is the acceptor Bohr radius, the Anderson-Mott insulator-to-metal transition occurs. However, a strong p-d antiferromagnetic interaction between the Mn and hole spin enhances strongly the acceptor binding energy and reduces $a_{B}$. It has been postulated [11] that owing to the large p-d interaction, the effect is particularly strong in nitrides, and may lead to the formation of a small polaron state, reminiscent of the Zhang-Rice singlet in high temperature superconductors.

In the presence of band carriers, the celebrated Ruderman-Kittel-Kasuya-Yosida (RKKY) mechanism of the spin-spin exchange interaction operates. In the context of III-V magnetic semiconductors, this mechanism was first discussed by Gummich and da Cunha Lima [12], and then applied to a variety of III-V and II-VI Mn-based layered structures [13].

It has been shown [5] that on the level of the meanfield and continuous medium approximations, the RKKY approach is equivalent to the Zener model. In terms of the latter, the equilibrium magnetization, and thus $T_{C}$ is determined by minimizing the Ginzburg-Landau free energy functional $F[\boldsymbol{M}(\boldsymbol{r})]$ of the system, where $\boldsymbol{M}(\boldsymbol{r})$ is the local magnetization of the localized spins [14,15]. This is a rather versatile approach, to which carrier correlation, confinement, $k \cdot p$ and spin-orbit couplings as well as weak disorder and antiferromagnetic interactions can be introduced in a controlled way, and within which the quantitative comparison of experimental and theoretical results is possible 
(Fig. 1). In the model, the hole contribution to $F$ is computed by diagonalizing the $6 \times 6$ Kohn-Luttinger $k \cdot p$ matrix containing the $\mathrm{p}-\mathrm{d}$ exchange contribution, and by subsequent computation of the partition function $Z, F_{c}=k_{B} T \ln Z$. The model is developed for p-type zinc-blende and wurzite semiconductors and allows for the presence of both biaxial strain and quantizing magnetic field. The enhancement of the tendency towards ferromagnetism by the carrier-carrier exchange interactions is described in the spirit of the Fermi liquid theory. Importantly, by evaluating $F_{c}(q)$, the magnetic stiffness can be determined, which together with magnetic anisotropy, yield the dispersion of spin waves [15] and the structure of magnetic domains [16]. Owing to a relatively small magnitudes of the s-d exchange coupling and density of states, the carrier-induced ferromagnetism is expected [5] and observed only under rather restricted conditions in ntype DMS [17,18].

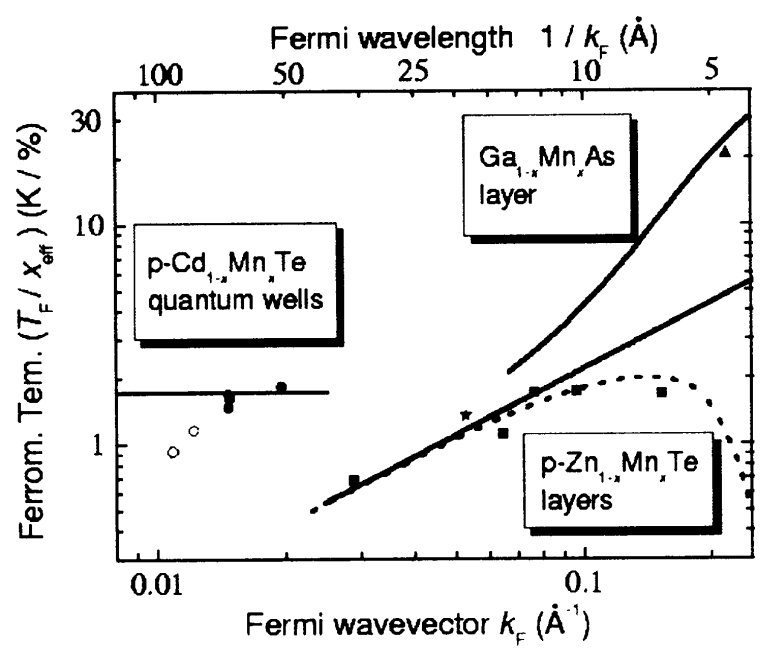

Figure 1. Ferromagnetic Curie temperature normalized by the $\mathrm{Mn}$ concentration vs. the Fermi wavector. Points are experimental and lines are theoretical (after $[6,7,14,21])$.

Since magnetic properties are controlled by the band carriers, the appealing possibility is to change the magnetic ordering isothermally, by light or by the electric field. Such tuning capabilities of the materials in question were put into the evidence in (In,Mn)As/(Al,Ga)Sb [19,20] and $(\mathrm{Cd}, \mathrm{Mn}) \mathrm{Te} /(\mathrm{Cd}, \mathrm{Zn}, \mathrm{Mg}) \mathrm{Te}[6,21]$ heterostructures, as shown in Figs. 2 and 3. Since the valence band is rather sensitive to strain, magnetic anisotropy (easy axis direction) can be manipulated by adjusting the lattice parameter of the substrate. According to theoretical results displayed in Fig. 4 , the crystallographic orientation of the easy axis at given strain, changes with the value of the valence band exchange splitting and, thus, with the temperature, as indeed observed [22].

In view of the promising properties of magnetic semiconductors, the development of a functional material with $T_{C}$ comfortably surpassing the room temperature, becomes an important challenge of today's materials science. A concentrated effort in this direction, stimulated by theoretical results recalled in Fig. 5, suggests that there is no fundamental limits precluding the achievement of this goal. However, solutions of problems associated with solubility of transition metals and electrical activity of impurities in various hosts are ahead.

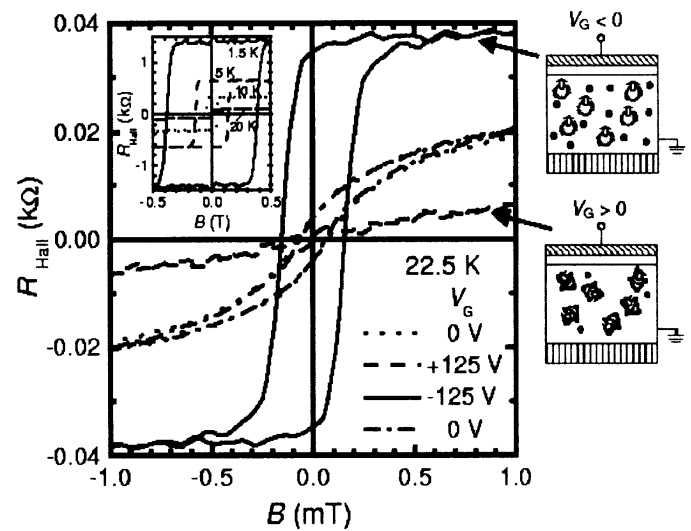

Figure 2. Magnetization hysteresis at various gate voltages evaluated by measurements of the anomalous Hall effect in FET structure of (In,Mn)As (after [20]).

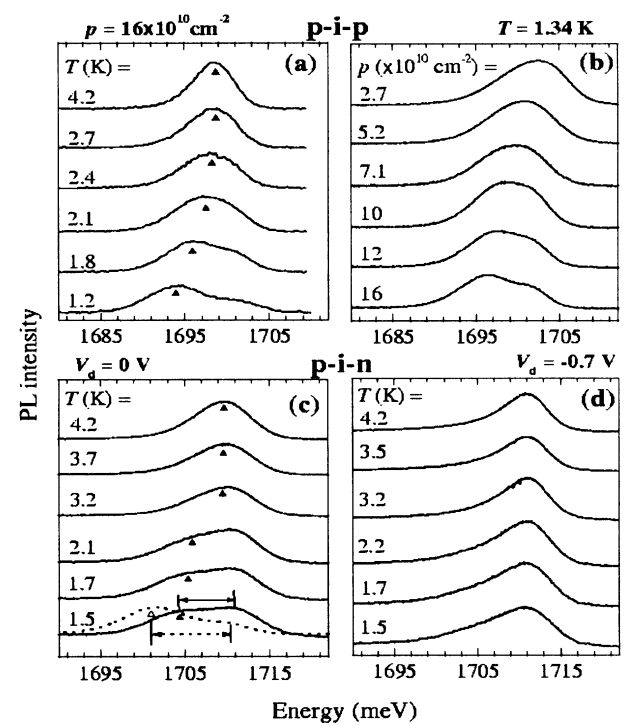

Figure 3. Effect of temperature (a,c,d), illumination (b) and bias voltage $(c, d)$ on photoluminescence line in quantum well of $(\mathrm{Cd}, \mathrm{Mn}) \mathrm{Te}$. Line splitting and shift witness the appearance of a ferromagnetic ordering (after [21]).

\section{Acknowledgements}

The author thanks his co-workers listed in the references for fruitful interactions. Author's research in Germany is supported by Alexander von Humboldt Foundation, while the work in Poland by State Committee for Scientific Research as well as by FENIKS and AMORE EC projects. 

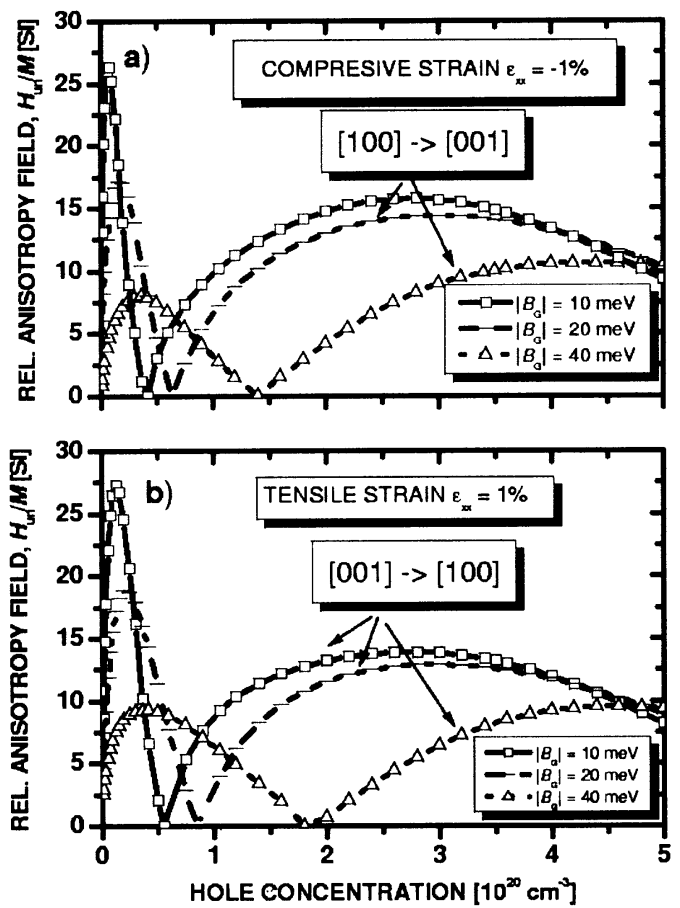

Figure 4. Computed anisotropy field for compressive (a) and tensile (b) strains for various value of the hole spin splitting parameter $B_{G}$ (after [14]).

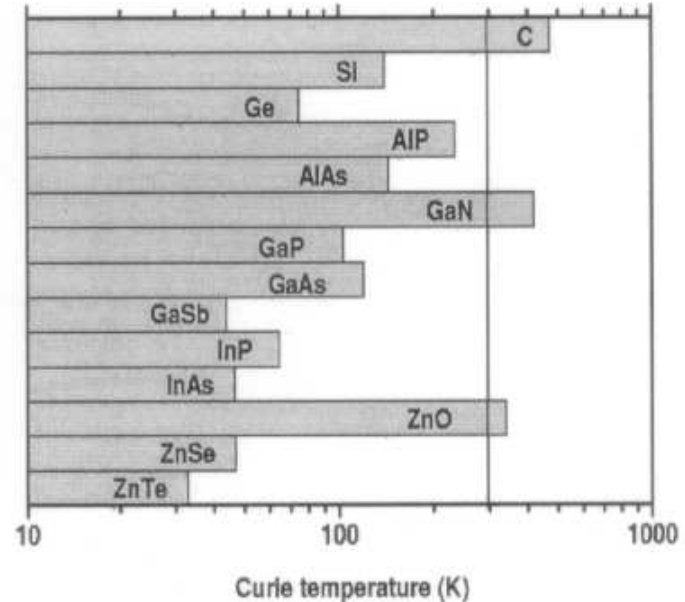

Figure 5. Computed Curie temperature for various materials containing $5 \%$ Mn per unit cell and $3.5 \times 10^{20}$ holes per $\mathrm{cm}^{3}$ (after [14]).

\section{References}

[1] H. Ohno, F. Matsukura, and Y. Ohno, JSAP International 5, 4 (2002). (www.jsapi.jsap.or.jp/); T. Dietl, Acta Phys. Polon. A 100 (Suppl.), 139 (2001), cond-mat/0201279; S. Wolf, D. D. Awshalom. R. A. Buhrman, J. M. Daughton, S. von Molnár, M. L. Roukes, A. Y. Chtchelkanova, and D. M. Treger, Science 294, 1488 (2001); special issue of Semicon. Sci. Technol. 17, No4 (2002), ed. H. Ohno.

[2] P. Wachter, Handbook on the Physics and Chemistry of Rare Earth, vol. 1 (Amsterdam: North-Holland, 1979) p 507; Y. Shapira, S. Foner, N.F. Oliveira, Jr.; and T.B. Reed, Phys. Rev. B 5, 2647 (1972).

[3] T. Story, R.R. Galazka, R.B. Frankel, P.A. Wolff, Phys. Rev. Lett. 56, 777 (1986).
[4] H. Ohno, H. Munekata, T. Penney, S. Von Molnár, L.L. Chang, Phys. Rev. Lett. 68, 2664 (1992); H. Ohno, A. Shen, F. Matsukura, A. Oiwa, A. Endo, S. Katsumoto, Y. Iye, Appl. Phys. Lett. 69, 363 (1996).

[5] T. Dietl, A. Haury, Y. Merle d'Aubigné, Phys. Rev. B 55, R3347 (1997).

[6] A. Haury, A. Wasiela, A. Arnoult, J. Cibert, S. Tatarenko, T. Dietl, Y. Merle d'Aubigné, Phys. Rev. Lett. 79, 511 (1997).

[7] D. Ferrand, J. Cibert, A. Wasiela, C. Bourgognon, S. Tatarenko, G. Fishman, T. Andrearczyk, J. Jaroszyński, S. Koleśnik, T. Dietl, B. Barbara, and D. Dufeu, Phys. Rev. B 63, 085201 (2001).

[8] H. Ohno, Science 281, 951 (1998); T. Dietl, Semicond. Sci. Technol. 17, 377 (2002); F. Matsukura, H. Ohno, T. Dietl; IIIV Ferromagnetic Semi-conductors, in: Handbook of Magnetic Materials, vol. 14, ed. K.H.J. Buschow (Elsevier, Amsterdam, 2002) pp. 1-87, and references therein.

[9] J. Szczytko, W. Bardyszewski, and A. Twardowski, Phys. Rev. B 64, 075306 (2001).

[10] O.M. Fedorych, E.M. Hankiewicz, Z. Wilamowski, J. Sadowski, Phys. Rev. B 66, 045201 (2002).

[11] T. Dietl, F. Matsukura, and H. Ohno, Phys. Rev. B 66, 033203 (2002).

[12] U. Gummich and I.C. da Cunha Lima, Solid State Commun. 76, 831 (1990).

[13] M.A. Boselli, A. Ghazali, and I.C. da Cunha Lima, J. Appl. Phys. 85, 5944 (1999); M.A. Boselli, A. Ghazali, and I.C. da Cunha Lima, Phys. Rev. B 62, 8895 (2000).

[14] T. Dietl, H. Ohno, F. Matsukura, J. Cibert, and D. Ferrand, Science 287, 1019 (2000); T. Dietl, H. Ohno, and F. Matsukura, Phys. Rev. B 63, 195205 (2001).

[15] J. König, T. Jungwirth, and A.H. MacDonald, Phys. Rev. B 64, 184423 (2001).

[16] T. Dietl, J. König, and A.H. MacDonald, Phys. Rev. B 64, 241201(R) (2001).

[17] T. Andrearczyk, J. Jaroszyński, M. Sawicki, Le Van Khoi, T. Dietl, D. Ferrand, C. Bourgognon, J. Cibert, S. Tatarenko, T. Fukumura, Z. Jin, H. Koinuma, and M. Kawasaki, in: Proceedings 25th Intl. Conf. on Physics of Semiconductors, Osaka, Japan, 2000, eds. N. Miura, T. Ando (Springer, Berlin, 2001) p. 235.

[18] J. Jaroszyński, T. Andrearczyk, G. Karczewski, J. Wróbel, T. Wojtowicz, E. Papis, E. Kamińska, A. Piotrowska, Dragana Popovic, and T. Dietl, Phys. Rev. Lett. 89, 266802 (2002).

[19] S. Koshihara, A. Oiwa, M. Hirasawa, S. Katsumoto, Y. Iye, C. Urano, H. Takagi, H. Munekata, Phys. Rev. Lett. 78, 4617 (1997).

[20] H. Ohno, D. Chiba, F. Matsukura, T. Omiya, E. Abe, T. Dietl, Y. Ohno, and K. Ohtani, Nature 408, 944 (2000).

[21] H. Boukari, P. Kossacki, M. Bertolini, J. Cibert, S. Tatarenko, D. Ferrand, A. Wasiela, J.A. Gaj, and T. Dietl, Phys. Rev. Lett. 88, 207204 (2002).

[22] M. Sawicki, F. Matsukura, A. Idziaszek, T. Dietl G.M. Schott, C. Ruester, G. Karczewski, G. Schmidt, L.W. Molenkamp, cond-mat/0212511. 